\begin{tabular}{|c|c|}
\hline Title & Optical pulse compression to $3.4 \mathrm{fs}$ in the monocy cle region by feedback phase compensation \\
\hline Author(s) & Y amane, Keisaku; Zhang, Zhigang; Oka, Kazuhiko; Morita, Ryuji; Y amashita, Mikio; Suguro, A kira \\
\hline Citation & $\begin{array}{l}\text { Optics Letters, 28(22), } 2258.2260 \\
\text { https://doi.org/10.1364/OL. } 28.002258\end{array}$ \\
\hline Issue Date & $2003-11-15$ \\
\hline Doc URL & http:/hdl.handle.net/2115/45338 \\
\hline Rights & (c) 2003 Optical Society of A merica \\
\hline Type & article \\
\hline File Information & OL28-22_2258-2260.pdf \\
\hline
\end{tabular}

Instructions for use 


\title{
Optical pulse compression to 3.4 fs in the monocycle region by feedback phase compensation
}

\author{
Keisaku Yamane, Zhigang Zhang, Kazuhiko Oka, Ryuji Morita, and Mikio Yamashita \\ Department of Applied Physics, Hokkaido University, Kita-13, Nishi-8, Kita-ku, Sapporo 060-8628, Japan
}

Akira Suguro

Technical Research Laboratory, Citizen Watch Company, Ltd., 840 Shimotomi, Tokorozawa 359-8511, Japan

Received June 2, 2003

\begin{abstract}
We compensated for chirp of optical pulses with an over-one-octave bandwidth (495-1090 nm; center wavelength of $655.4 \mathrm{~nm}$ ) produced by self-phase modulation in a single argon-filled hollow fiber and generated 3.4 -fs, 1.56 optical-cycle pulses $(500 \mathrm{~nJ}, 1-\mathrm{kHz}$ repetition rate). This was achieved with a feedback system combined with only one $4-f$ phase compensator with a spatial light modulator and a significantly improved phase characterizer based on modified spectral phase interferometry for direct electric-field reconstruction. To the best of our knowledge, this is the shortest pulse in the visible-to-infrared region. (C) 2003 Optical Society of America

OCIS codes: $320.5520,320.7100$.
\end{abstract}

The generation of sub-5-fs optical pulses was achieved in recent years by several groups. ${ }^{1-4}$ In 2002 , a 4 -fs pulse compression in noncollinear optical parametric amplifiers with a bandwidth from 480 to $750 \mathrm{~nm}$ was reported. ${ }^{4}$ However, further compression is limited by the difficulty in ultrabroadband phase matching and compensation of a high-order spatial chirp and the filter effect of a nonlinear crystal in the secondharmonic generation (SHG) frequency-resolved optical gating measurement.

Key technologies to realize monocycle pulses are the generation of over-one-octave ultrabroadband pulses, their phase and amplitude characterization and precise phase compensation. Although the spectral phase interferometry for direct electric-field reconstruction (SPIDER) technique has the potential of characterizing the spectral phase that extends to over one octave,${ }^{5}$ it has low sensitivity for the measurement of such ultrabroadband (low spectral density) signals. We developed a modified SPIDER (M-SPIDER) technique that greatly improved its sensitivity. ${ }^{6,7}$ It is difficult to compress broadband optical pulses with an over-one-octave bandwidth by use of conventional passive chirped compensators such as prism and (or) grating combinations, chirped mirrors, because of their bandwidth limitation and high-order dispersion. A liquid-crystal spatial light modulator (SLM) is a superior candidate for a programmable phase compensator of over-one-octave pulses. It has a transmission spectrum from 300 to $1500 \mathrm{~nm}$. A deformable mirror ${ }^{4}$ is another candidate but it has the disadvantages of being cumbersome, imperfect phase calibration of the membrane deflection, low spatial and phase resolutions, and high deflection losses. We previously demonstrated that a SLM phase compensator enables us to compress pulses to sub-5 fs by inputting individual orders of dispersion into a SLM. ${ }^{1}$ In this Letter we report, for the first time to the best of our knowledge, a 1.56-optical-cycle, 3.4-fs pulse generation through a single hollow fiber and only a programmable SLM by phase feedback with a significantly improved M-SPIDER.

The experimental setup is shown in Fig. 1 . The output beam from a Ti:sapphire laser amplifier system ( $<30$-fs pulse duration, $\sim 790$-nm center wavelength, $1-\mathrm{kHz}$ repetition rate) was divided into two beams with a beam splitter. One beam with a pulse energy of $94 \mu \mathrm{J}$ was coupled into a hollow fiber with a $34-\mathrm{cm}$ length and a $100-\mu \mathrm{m}$ inner diameter, which was positioned in a chamber filled with argon (3.0 atm). The chamber had two 1-mm-thick fused-silica windows. The other beam with a pulse energy of $2.4 \mu \mathrm{J}$ was sent into the M-SPIDER as an external reference beam. After passing through the hollow fiber, pulses



Fig. 1. Experimental setup: CM1, CM2, Al-coated concave mirrors; G1, G2, Al-coated reflective gratings; PS, periscopes; L1, L2, fused-silica focusing lenses $(f=50 \mathrm{~mm})$; HF, Ar-filled hollow fiber; FM, flip mirrors; BS1, BS2, wedged beam splitters; FL, optical filter (cutoff of $500 \mathrm{~nm}$ ); FRAC, fringe-resolved autocorrelator; GDD, group delay dispersion glass. 
with $16-\mu \mathrm{J}$ energy were injected into an active phase compensator that contained a specifically designed liquid-crystal SLM (Citizen Watch Co., pixel number of 648 , pixel width of $97 \mu \mathrm{m}$, gray-scale resolution of 192), two aluminum-coated gratings (blaze wavelength of $500 \mathrm{~nm}$, groove density of 150 lines $/ \mathrm{mm}$ ), and two concave mirrors (focal length of $350 \mathrm{~mm}$ ). The output pulse $(0.5-\mu \mathrm{J}$ energy) from the phase compensator was injected into the M-SPIDER.

In the M-SPIDER, the two replicas with delay time $\tau$ were produced by a Michelson interferometer. The replicas were upconverted with the external reference chirped pulse, which passed through a 10-cm-long TF5 glass twice, in a $20-\mu \mathrm{m}$-thick $\beta$-barium borate crystal (BBO type II, $45^{\circ}$ ). The interferogram with a spectral shear $\Omega$ was measured by a spectrometer (Chromex 500is, 1200 lines $/ \mathrm{mm}$ ) with an intensified chargecoupled device (ICCD, Andor Technology DH520, $1024 \times 256$ pixels). The synchronously gated ICCD was cooled to $-25{ }^{\circ} \mathrm{C}$ to increase the signal-to-noise ratio.

The essential points for successful feedback are accurate measurement and accurate phase compensation, for which we exerted much effort. To measure phase $\phi(\omega)$ accurately, we analyzed the phase errors invoked by the inaccurately measured $\tau+\delta \tau$ and $\Omega+\Delta \Omega$. We found that error $\delta \tau$ would introduce an additional second-order dispersion $\delta \tau \omega^{2} / 2 \Omega$, and the error $\Omega+$ $\Delta \Omega$ would result only in an amplitude error of the phase $[(1-\Delta \Omega / \Omega) \phi(\omega)]$. Therefore we concentrated mainly on increasing the accuracy of $\tau$.

In the SPIDER measurement, the derived phase difference contains a delay-dependent linear term $\omega \tau$ according to $\theta_{\text {SPIDER }}(\omega)=\phi(\omega)-\phi(\omega-\Omega)+\omega \tau$. This term can be removed by measurement of the delay of the two replica pulse pairs. However, as suggested by Iaconis and Walmsley, ${ }^{5}$ if there is a system error in the Michelson interferometer, it would be best to measure the SPIDER signal for $\Omega=0$ and subtract it as the background. This can be achieved by measurement of the second-harmonic (SH) interferogram since $\theta_{\mathrm{SH}}(\omega)=\omega \tau$.

The crystal for sum-frequency generation in the SPIDER is cut for a type II phase-matching condition, which is insensitive to the bandwidth limitation, whereas the SHG is achievable with type I phase matching. Realignment of the crystal, the lens, the detector, and the slit is required for the best signal intensity. This alignment does not correspond to the same conditions as measurement of the sumfrequency waves of the SPIDER. To avoid perturbation of the SPIDER measurement and to obtain a reproducible signal, we built a separate optical path for the independent alignment and measurement of the SH interferogram (Fig. 1), which permitted highly reproducible measurements.

Next we examined the error source in the previous system $^{6,7}$ and made some important modifications as described below. First, we improved the beam splitters in the Michelson interferometer. We found that the SPIDER interferogram was spoiled by the interference between front and back surface reflections of the beam splitters. An antireflection coating on the backside should avoid this, however, it is difficult to make an antireflection coating for an over-oneoctave bandwidth. Therefore, we replaced them with wedged beam splitters with a thickness of $0.5 \mathrm{~mm}$ and a wedged angle of $0.5^{\circ}$ (20\% reflection and $80 \%$ transmission for $s$ polarization from 400 to $1300 \mathrm{~nm}$ and fused-silica substrates). Consequently, the backreflection interference is completely eliminated.

Second, we recalibrated the spectrometer using a mercury lamp (less than $0.05 \mathrm{~nm}$ over the whole spectral range $300-460 \mathrm{~nm}$ ) at a sufficiently high accuracy. However, we found that the calibrated wavelength contains a nonlinear error that produces a small but not negligible distortion in measured spectral interference fringe spacing. This error could cause an error in delay time $\tau$ of the order of a few femtoseconds that would result in a group delay dispersion error of several tens of femtoseconds squared. This error makes subtracting $\omega \tau$ from the measured phase difference more crucial, rather than just determining delay time $\tau$ through the measured fringe period.

Third, we significantly increased the SH signal intensity. The intensity of the $\mathrm{SH}$ had been weak so far, and it had been difficult to measure over the same wavelength range as in the SPIDER measurement. The input pulse has a bandwidth from 490 to $1100 \mathrm{~nm}$, corresponding to a SH signal from 245 to $550 \mathrm{~nm}$. On the other hand, the SPIDER signal has a bandwidth of $300-460 \mathrm{~nm}$. The $\mathrm{SH}$ signal is too broad to cover the SPIDER wavelength range. Therefore, we initially reduced the pressure of Ar gas from 3.0 to $0.8 \mathrm{~atm}$ so that the spectral range of the intensified SH interferogram has approximately the same wavelength range as in the SPIDER [Fig. 2(c)].

Fourth, since the pulse has an over-one-octave bandwidth, the first-order diffraction of the gratings can overlap with the second-order diffraction on the SLM. In the 4- $f$ system, the second-order diffraction can be modulated unexpectedly by the SLM and can reduce the accuracy of phase compensation. We found that the proper choice of a blaze wavelength could reduce the influence of the second-order diffraction on the output from the compensator. For a spectrum of $490-1100-\mathrm{nm}$ bandwidth, if the blaze wavelength is

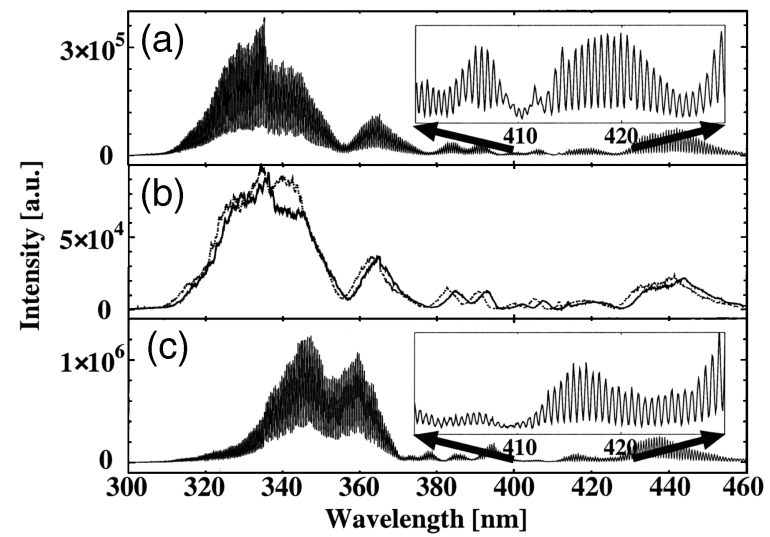

Fig. 2. Results of modified SPIDER measurements: (a) M-SPIDER signal, (b) replicas, (c) the $\mathrm{SH}$ interferogram. 


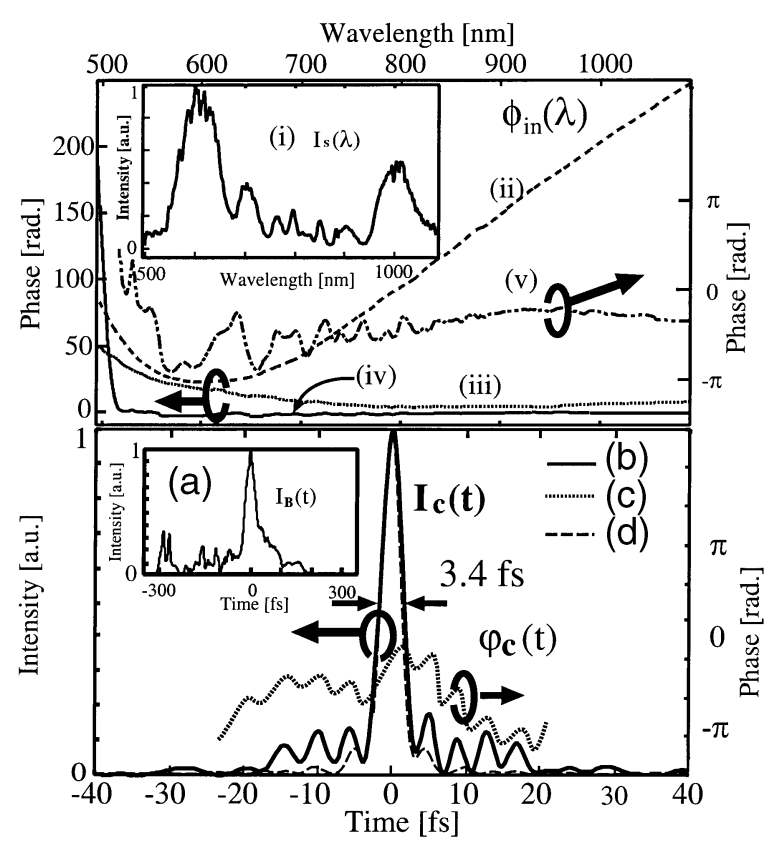

Fig. 3. Results of feedback compensation: (i) intensity spectrum, (ii) spectral phase before compensation. Spectral phases after (iii) first and (iv) second feedbacks. (v) detail of (iv). (a) Temporal intensity before compensation, (b) intensity and (c) phase after second feedback, (d) transform-limited pulses.

chosen to be $500 \mathrm{~nm}$, the intensity of the second-order diffraction can be reduced to only a few percent of the first order. In addition, use of the longer focal length (from $f=200 \mathrm{~mm}$ in Ref. 1 to $f=350 \mathrm{~mm}$ ) of two concave mirrors reduced the loaded bandwidth of each pixel (from $\Delta \lambda=3.0 \mathrm{~nm}$ to $\Delta \lambda=1.9 \mathrm{~nm}$ ) and hence the load of phase compensation per each pixel.

Finally, phase compensation with multiple feedbacks is in great demand, especially when spectral shear $\Omega$ for each frequency component cannot be regarded as a constant. This could happen when the input pulse has a broader temporal width than the necessary $10 \%$ of the reference pulse width. ${ }^{5}$ The first feedback could make the input pulse narrow down such that spectral shear $\Omega$ could be taken as a constant. Then the second feedback with such shear could remove the residual errors. In general, $n$-multiple feedbacks rapidly decrease the insufficiency of phase compensation, which is due to any errors $\Delta \Omega$ of the spectral shear, according to $\phi^{(n)}(\omega)=\left|(\Delta \Omega /(\Omega+\Delta \Omega))^{n}\right| \phi^{(0)}(\omega)$, where $\phi^{(0)}(\omega)$ and $\phi^{(n)}(\omega)$ are the phases after and before $n$-times feedback compensation, respectively.

On the basis of the above improvement, we conducted a pulse compression experiment. First we measured the $\mathrm{SH}$ interferogram [Fig. 2(c)] and then after a few minutes the SPIDER interferogram
[Fig. 2(a)]. Because the pulses have broad bandwidths, it was necessary for us to obtain the signals by five rotations of the grating in the spectrometer, which were automatically controlled (exposure time was $0.1 \mathrm{~s} \times 100$ ). We found that the replicas became similar over the whole range from 300 to $460 \mathrm{~nm}$ [Fig. 2(b)].

Figure 3, curve (i) is the measured spectrum of the pulse output from the phase compensator; curve (ii) represents the retrieved phase before feedback. This negative phase is fed back to the SLM, and the resultant reconstructed phase is curve (iii). After two feedback compensations, the spectral phase was almost flat [Fig. 3, curve (iv), the enlarged version is curve (v)], which demonstrates that the feedback pulse compression is powerful. The $\tau$ and $\Omega / 2 \pi$ confirmed in this experiment were $935 \mathrm{fs}$ and $4.12 \mathrm{THz}$, respectively. The retrieved temporal intensity and phase of compensated pulses are shown in Fig. 3 as curves (b) and (c); Fig. 3(a) shows pulses before compensation. We found that sidelobes were almost eliminated and the pulse duration was $3.4 \mathrm{fs}$, close to the $3.0-\mathrm{fs}$ transform-limited pulse with small sidelobes [Fig. 3(d)]. This pulse duration corresponds to only 1.56 cycle for a central wavelength of $655.4 \mathrm{~nm}$. This phase compensation was also confirmed by an independent measurement of interferometric autocorrelation traces under the bandwidth limitation of $500-980 \mathrm{~nm}$ by a slit on the SLM.

In conclusion, we have demonstrated the successful 3.4-fs, 1.56-optical-cycle pulse compression with negative phase feedback into only a SLM phase compensator. The correct phase measurement with the M-SPIDER for over-one-octave pulses was achieved with the improved hardware and the retrieval process.

K. Yamane's e-mail address is k-yamane@ eng.hokudai.ac.jp.

\section{References}

1. N. Karasawa, L. Li, A. Suguro, H. Shigekawa, R. Morita, and M. Yamashita, J. Opt. Soc. Am. B 18, 1742 (2001).

2. A. Cheng, G. Tempea, T. Brabec, K. Ferencz, and F. Krausz, in Ultrafast Phenomena XI (Springer-Verlag, Berlin, 1998), p. 8.

3. A. Baltuška, M. S. Pshenichnikov, and D. A. Weiersma, IEEE J. Quantum Electron. 35, 459 (1999).

4. A. Baltuška, T. Fuji, and T. Kobayashi, Opt. Lett. 27, 306 (2002).

5. C. Iaconis and I. A. Walmsley, IEEE J. Quantum Electron. 35, 501 (1999).

6. M. Hirasawa, N. Nakagawa, K. Yamamoto, R. Morita, H. Shigekawa, and M. Yamashita, Appl. Phys. B 74, S225 (2002).

7. R. Morita, M. Hirasawa, N. Karasawa, S. Kusaka, N. Nakagawa, K. Yamane, L. Li, A. Suguro, and M. Yamashita, Meas. Sci. Technol. 13, 1710 (2002). 